\title{
COMPARAÇÃO DAS RESERVAS GLICOGÊNICAS EM RATOS JOVENS E ENVELHECIDOS TRATADOS COM PICOLINATO DE CROMO
}

\author{
GLYCOGEN RESERVES IN YOUNG AND AGED RATS TREATED WITH CHROMIUM PICOLINATE \\ RESERVAS DE GLUCÓGENO EN RATAS JÓVENES Y ENVEJECIDAS TRATADAS CON \\ PICOLINATO DE CROMO
}

\begin{abstract}
Marcella Damas Rodrigues ${ }^{1}$ (Fisioterapeuta)

Sérgio Henrique Borin

(Fisioterapeuta)

Patrícia Carla Paulino (Bióloga)

Eder João Arruda' (Fisioterapeuta)

Carlos Alberto da Silva' (Biólogo)

1. Universidade Metodista de Piracicaba (UNIMPP), Piracicaba, SP, Brasil.
\end{abstract}

\section{Correspondência:}

Rua Rossini Pinto, 117 - Jardim

Panorama. 13416-784, Piracicaba,

SP, Brasil.

marcella.damas@yahoo.com.br

\section{RESUMO}

Introdução: Entre os suplementos utilizados no meio esportivo, o mineral cromo tem se destacado, principalmente por potencializar a ação da via insulínica, ação extremamente importante na manutenção da homeostasia metabólica. A ação do cromo parece ter ação importante como coadjuvante nas dinâmicas da ação insulínica. Objetivo: Avaliar o perfil glicogênico, bem como a sensibilidade tecidual à insulina e a pancreática à glicose em ratos jovens e envelhecidos tratados com picolinato de cromo. Método: Foram utilizados ratos Wistar, com idade de 3 e 24 meses, distribuídos em quatro grupos experimentais $(n=6)$, assim denominados: jovens $(J)$, jovens suplementados com picolinato de cromo (JP, $80 \mu \mathrm{g} / \mathrm{Kg}$ ), envelhecidos (E) e envelhecidos suplementados com picolinato de cromo (EP, $80 \mu \mathrm{g} / \mathrm{Kg})$. A sensibilidade à insulina foi avaliada através do teste de tolerância à insulina (ITT, $2 \mathrm{U} / \mathrm{Kg}$ ) e a sensibilidade pancreática, através do teste de tolerância à glicose (GTT, 2 g/Kg). Na análise estatística foi utilizado teste de normalidade de dados de Kolmogorov-Smirnov, seguido de ANOVA e o teste post-hoc de Tukey, $p<0,05$. Resultados: $O$ grupo envelhecido apresentou menores reservas glicogênicas se comparado ao grupo jovem; por sua vez, o tratamento com picolinato promoveu elevação das reservas hepáticas de ratos jovens sem efeito nos envelhecidos. No mesmo perfil de análise, foi demonstrado que o tratamento com picolinato promoveu elevação das reservas glicogênicas musculares, efeito observado tanto nos jovens quanto nos envelhecidos. No grupo jovem, não foi observada diferença no ITT, porém houve redução da área sob a curva descrita no GTT. No grupo envelhecido, houve elevação da responsividade à insulina no ITT e redução da área sob a curva. Conclusão: O picolinato expressou ação de secretagogo e sensibilizador da ação insulínica, com expressão mais significativa nos músculos envelhecidos.

Palavras-chave: metabolismo, envelhecimento, glicogênio.

\begin{abstract}
Introduction: Among the supplements used in sports, the mineral chromium has stood out particularly by enhancing the action of insulin route, action extremely important in maintaining metabolic homeostasis. The chromium action seems to have significant action as an adjunct in the dynamics of insulin action. Objective: To evaluate the glycogen profile and the tissue sensitivity to insulin and pancreatic glucose sensitivity in young and aged rats treated with chromium picolinate. Methods: Wistar rats were used, aged 3 and 24 months, divided into four experimental groups $(n=6)$, so named:young ( $Y$, young supplemented with chromium picolinate $(Y P, 80 \mu \mathrm{g} / \mathrm{kg})$, aged (A) and aged supplemented with chromium picolinate (AP, $80 \mu \mathrm{g} / \mathrm{kg}$ ). The insulin sensitivity was assessed by the insulin tolerance test (ITT, $2 U / \mathrm{Kg}$ ) and pancreatic sensitivity was assessed by the glucose tolerance test (GTT, $2 \mathrm{~g} / \mathrm{Kg})$. Statistical analysis used Kolmogorov-Smirnov Test for Normality, followed by ANOVA and Tukey's Post-hoc test, $p<0.05$. Results: The aged group showed lower glycogen reserves compared to the young group; in turn, treatment with chromium picolinate causes an increase in liver reserves of young rats with no effect on aged rats. At the same profile analysis, it was shown that treatment with chromium picolinate promoted an increase in muscle glycogen reserves, effect observed in both young and aged rats. In the young group, no difference was observed in the ITT, but there was a decrease of the area under the curve described in GTT. In the aged group, there was an increase in responsiveness to insulin in the ITT and decrease of the area under the curve. Conclusion: The chromium picolinate expressed secretagogue and sensitizer action of insulin action with most significant expression in aging muscles.
\end{abstract}

Keywords: metabolism, aging, glycogen.

\section{RESUMEN}

Introducción: Entre los suplementos que se usan en los deportes, el mineral cromo ha sido distinguido, en particular por el aumento de la acción de la vía de la insulina, una acción muy importante en el mantenimiento de la homeostasis metabólica. La acción del cromo parece tener una acción significativa como coadyuvante en la dinámica de la acción de la insulina. Objetivo: Evaluar el perfil del glucógeno y la sensibilidad de los tejidos a la insulina y de la glucosa pancreática en ratas jóvenes y envejecidas tratadas con picolinato de cromo. 
Métodos: Se utilizaron ratas Wistar con edades de 3 y 24 meses, divididas en cuatro grupos experimentales ( $n=6$ ), Ilamados asi: jóvenes (Y), jóvenes complementados con picolinato de cromo (JP, $80 \mathrm{mg} / \mathrm{Kg}$.), envejecidos (E) y envejecidos suplementados con picolinato de cromo (EP $80 \mathrm{mg} / \mathrm{Kg}$.). La sensibilidad a la insulina se evaluó mediante la prueba de tolerancia a la insulina (ITT, 2 U/Kg.) y la sensibilidad pancreática, mediante la prueba de tolerancia a la glucosa (GTT, $2 \mathrm{~g} / \mathrm{Kg}$.). En el análisis estadístico se utilizó la prueba de normalidad de Kolmogorov-Smirnov seguida por ANOVA y la prueba post-hoc de Tukey, $p<0,05$. Resultados: El grupo de ratas envejecidas mostró reservas de glucógeno más bajas en comparación con el grupo de jóvenes; a su vez, el tratamiento con picolinato de cromo causó la elevación de las reservas hepáticas sin efecto en ratas jóvenes. Al mismo análisis de perfiles, se demostró que el tratamiento con picolinato de cromo promovió un aumento de las reservas de glucógeno muscular, efecto observado en ratas jóvenes y envejecidas. En el grupo joven, no se observó ninguna diferencia en la ITT, pero hubo una reducción en el área bajo la curva en la GTT. En el grupo envejecido, hubo aumento en la capacidad de respuesta a la insulina en la ITT y reducción en el área bajo la curva. Conclusión: El picolinato expresó una acción secretagoga y sensibilizadora de la acción de la insulina, con la expresión más significativa en los músculos envejecidos.

Palabras clave: metabolismo, envejecimiento, glucógeno.

\section{INTRODUÇÃO}

A plasticidade histofisiológica das fibras musculares esqueléticas permite que estas sejam capazes de adaptar constantemente sua funcionalidade de acordo com as necessidades funcionais ou metabólicas deflagrando mudanças no seu tamanho, no tipo de fibra ou no perfil de utilização de substratos metabolizáveis'.

Com relação à hipotrofia muscular induzida por desuso tem sido descrito uma série de efeitos deletérios, tais como a redução da densidade dos capilares, dos sarcômeros em série, da força e resistência à fadiga ${ }^{2}$. Segundo Hilder et al. ${ }^{3}$, dentro do perfil metabólico, o desuso muscular crônico e a imobilização são condições que diminuem a eficiência das vias sinalizadoras de insulina, induzindo o quadro de resistência insulínica. Há de se considerar que a hipotrofia é um processo que apresenta características específicas para cada tipo de músculo e têm-se demonstrado que o músculo sóleo é o mais comprometido em situações de desuso por ser predominantemente formado por fibras do tipo I, antigravitacionário e possuir menor adaptação em relação às fibras do tipo 11.

As fibras lentas apresentam ainda marcantes alterações histofisiológicas como irregularidades no retículo sarcoplasmático, fibrilas desintegradas, lesão mitocondrial, linhas Z estendidas, como condensação e fragmentação da cromatina nuclear e redução de sarcômeros em paralelo e em série na condição de desuso ${ }^{4}$.

Concomitante ao envelhecimento, diversas funções fisiológicas são prejudicadas, com destaque às relacionadas com a função muscular, que se apresenta diminuída, afetando significativamente a qualidade de vida ${ }^{5,6}$. A perda da massa muscular e consequentemente da força muscular é um indicativo marcante da perda da mobilidade e da capacidade funcional do indivíduo que está envelhecendo 7 . Sabe-se que a força muscular atinge seu pico por volta dos trinta anos de idade e é preservada por volta dos cinquenta anos. A partir dessa idade, a taxa de progressão de redução da força se dá em torno de 8 a 15\% por década e, diante do decréscimo no número de fibras musculares a atrofia da fibra tipo II é a mais acometida ${ }^{8}$. Essas alterações podem levar à perda no desempenho de habilidades motoras, sejam elas relacionadas ao desempenho atlético de alto nível ou à vida funcional diária9.

Dentre os suplementos utilizados no meio esportivo, destaca-se o mineral Cromo, cuja função primária é potencializar os efeitos da insulina ${ }^{10}$. Também aumenta a fluidez da membrana celular para facilitar a ligação da insulina com seu receptor, ao qual o GTF (fator auxiliar de transporte de glicose da via insulínica) funciona como um carreador para proteínas celulares deficientes em cromo ${ }^{11}$. Mais recentemente, o Cromo foi caracterizado como componente participante do mecanismo de amplificação da sinalização celular de insulina, ou seja, um fator colaborador do aumento da sensibilidade de receptores insulínicos na membrana plasmática ${ }^{12}$.

A sua suplementação tem sido utilizada com a finalidade de promover aumento de massa muscular e diminuição da gordura corporal via ação glicogênica e sensibilidade insulínica; todavia, há escassez de evidências científicas comprovando esses possíveis efeitos positivos da suplementação com cromo ${ }^{13}$.

Avaliar o perfil glicogênico, bem como a sensibilidade tecidual á insulina e pancreática ao bolus de glicose em ratos jovens e envelhecidos tratados com picolinato de cromo.

\section{MATERIAL E MÉTODOS}

Foram utilizados ratos albinos Wistar com idade de 3 e 24 meses de idade, pesando entre 180-220g adquiridos junto à empresa ANILABPaulínia, SP, Brasil. Os animais foram alimentados com ração e água ad libitum, sendo submetidos a ciclo foto periódicos de 12h claro/escuro e distribuídos em quatro grupos experimentais ( $n=6)$, assim denominados: jovens (J), jovens suplementados com picolinato de cromo (JP), envelhecidos (E) e envelhecidos suplementados com picolinato de cromo (EP). O trabalho foi aprovado pelo Comitê de Ética UA - UNIMEP sob protocolo 09/2012.

O tratamento consistiu da administração do picolinato de cromo na dose de $80 \mu \mathrm{g} / \mathrm{Kg}$ disponível na água para beber ${ }^{14}$. Optou-se pelo picolinato de cromo, pois esta forma não é tóxica ao organismo, bem como é a forma encontrada em muitos alimentos. Após anestesia com pentobarbital sódico $(40 \mathrm{mg} / \mathrm{kg}$, i.p), os animais foram sacrificados por deslocamento cervical e amostras do fígado e dos músculos sóleo, gastrocnêmio porção branca, gastrocnêmio porção mista, foram retirados e encaminhados para determinação das reservas glicogênicas segundo o método do fenol sulfúrico $^{15}$. A sensibilidade à insulina foi avaliada através do teste de tolerância a insulina (ITT) bem como a sensibilidade pancreática foi avaliada através do teste de tolerância à glicose (GTT), segundo Rafacho et al. ${ }^{16}$. Na análise do ITT os animais foram anestesiados com pentobarbital sódico (40 mg/Kg, ip) e através de uma corte na cauda, a glicemia foi avaliada com o uso de glucometer (tempo zero) e a seguir insulina $(2 \mathrm{U} / \mathrm{Kg}$ ) foi injetada intraperitoneal 
e a glicemia novamente avaliada nos tempos 2, 5; 5; 10; 15; 20; 25 e 30min. A velocidade de decaimento foi calculada através com o software Origin ${ }^{\circledR}$. No GTT, o mesmo procedimento anestésico foi utilizado, no entanto, após o tempo zero, foi injetado glicose (2 mg/ $\mathrm{Kg}$ ) e amostras de sangue coletadas nos tempos 15, 30, 45, 60, 90 e 120 min e a glicemia avaliada com o mesmo método.

Na avaliação estatística foi utilizado o teste de normalidade (Kolmogorov-Smirnov) seguido de ANOVA e teste de Tukey, com nível de significância de 5\%. Esta análise foi realizada no software ORIGIN (versão 5.0).

\section{RESULTADOS}

Inicialmente foram avaliadas as reservas glicogênicas hepáticas na presença do picolinato de cromo sendo observado que, no grupo jovem, o conteúdo foi elevado em 42\%, fato que não ocorreu no grupo envelhecido (figura 1). No mesmo sentido de análise, foi observado o mesmo efeito nas reservas glicogênicas musculares onde foram verificadas reservas 20\%, 15\% e 15,6\% maiores nos músculos sóleo, gastrocnêmio porção branca e gastrocnêmio porção vermelha, respectivamente (figura 2).

No que se refere ao grupo envelhecido não tratado, foi observado que suas reservas estavam menores se comparado ao grupo jovem, uma vez que, as reservas do sóleo foram 44,6\% menores se comparado ao jovem, efeito também observado nos demais músculos, onde foram constatadas reservas 35,8\% e 28\% no gastrocnêmio porção branca e gastrocnêmio porção vermelha, respectivamente (figura 2).

A avaliação das reservas glicogênicas do tecido muscular de ratos envelhecidos tratados com o picolinato de cromo mostrou elevação no conteúdo, atingindo valores de 46\%, 28\% e 17\% maiores respectivamente no sóleo, gastrocnêmio porção branca e gastrocnêmio porção vermelha (figura 2).

A constatação de mudanças nas reservas glicogênicas instigou a busca pelo possível sítio de ação do cromo, iniciando pelo grupo jovem. Neste sentido, não foi observado diferença na constante de decaimento da glicemia no grupo jovem, submetido ao ITT (figura 3). Por outro lado, foi significativa a redução na área sob a curva demonstrada no teste de tolerância a glicose, o qual foi 37,2\% menor no grupo jovem tratado, indicando ação secretagogo do cromo (figura 4).

A análise do ITT do grupo envelhecido tratado com picolinato de cromo mostrou que o mineral promoveu elevação na responsividade à insulina, elevando em 99,7\% a velocidade de decaimento da glicemia. Por sua vez, a avaliação da resposta pancreática mostrou que o picolinato de cromo sensibilizou as células beta pancreáticas reduzindo em $15 \%$ a área sob a curva, como pode ser verificado no GTT (figura 4).

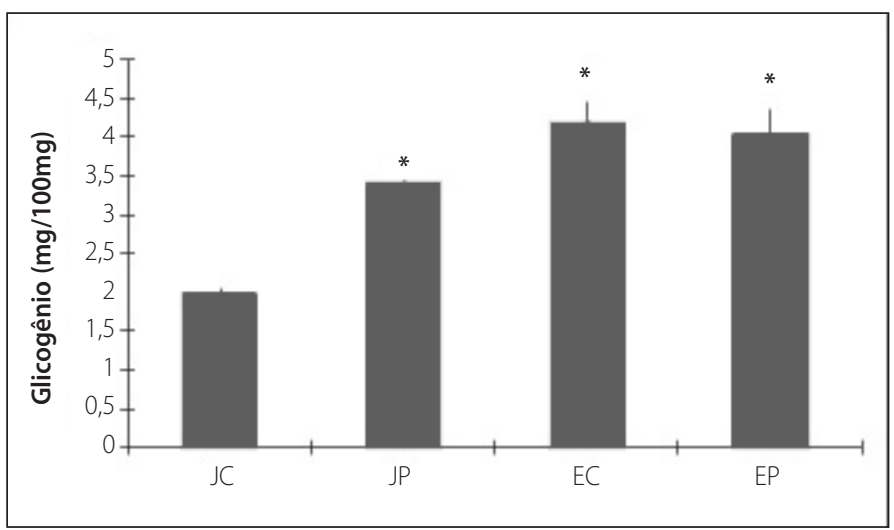

Figura 1. Concentração hepática de glicogênio (mg/100mg) de ratos Jovens (J) e envelhecidos (E) nas condições controle (C) e tratados com Picolinato de Cromo (P). Os valores correspondem à média \pm epm, $n=5 .{ }^{*} p<0,05$ comparado ao controle.

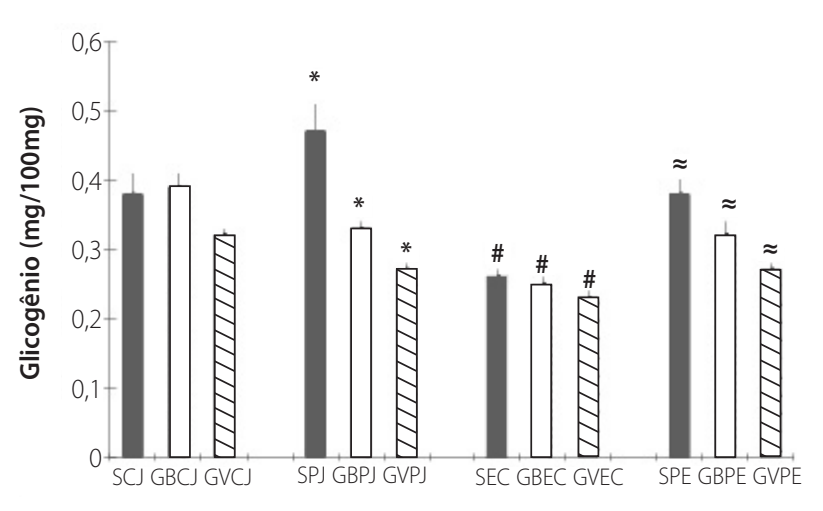

Figura 2. Concentração de glicogênio (mg/100mg) dos músculos Sóleo (S), Gastrocnêmio Porção Branca (GB) e Gastrocnêmio Porção Vermelha (GV) de ratos Jovens (J) e Envelhecidos (E) condições controle (C) e tratados com Picolinato de Cromo (P). Os valores correspondem à média \pm epm, $n=5$. $P<0,01$ comparado ao controle, $\# p<0,05$ comprado ao jovem, $\approx p<0,05$ comparado ao envelhecido não tratado.

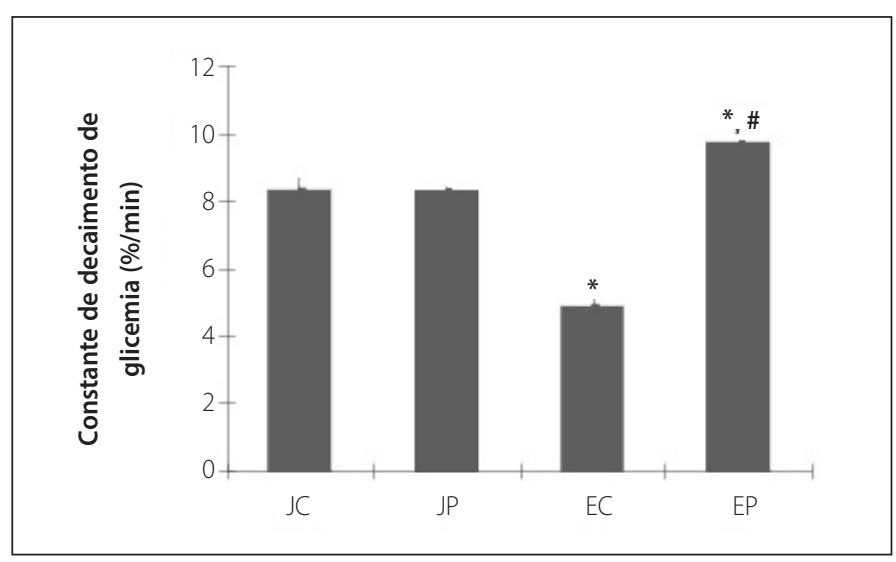

Figura 3. Constante de decaimento da glicemia (KITT; \%/min) de ratos Jovens (J) e Envelhecidos (E) controle (C) e tratados com Picolinato de Cromo (P). Os valores correspondem á média \pm epm, $n=5 .^{*} p<0,05$ comparado ao controle; $\# p<0,05$ comparando o grupo envelhecido tratado com o envelhecido não tratado.

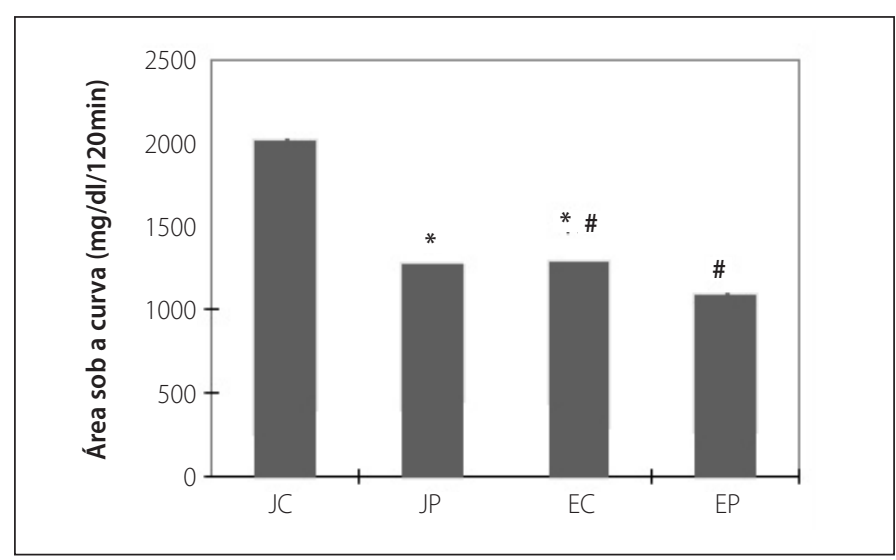

Figura 4. Área sob a Curva (mg/dL/120 min) de ratos Jovens $(J)$ e Envelhecidos (E) controle $(C)$ e tratados com Picolinato de Cromo (P). Os valores correspondem à média \pm epm, $n=5$. * $\mathrm{p}<0,05$ comparado ao controle; $\# \mathrm{p}<0,05$ comparando enveIhecido tratado ao envelhecido tratado.

\section{DISCUSSÃO}

A homeostase energética depende da disponibilidade de substratos metabolizáveis, bem como da funcionalidade das vias bioquímicas que permitem tanto a formação quanto a mobilização das reservas. Dentre os principais sistemas que comandam o equilíbrio metabólico, o fígado e os músculos merecem destaque, uma vez que, constituem 
os principais reservatórios e são sensíveis tanto a insulina, hormônio relacionado à formação das reservas, quanto aos hormônios contrarreguladores, os quais promovem a mobilização ${ }^{17}$.

De acordo com o estudo de Leto e Satie ${ }^{18}$, a insulina controla o metabolismo dos carboidratos, de modo que interage com receptores das células alvo, evento este que desencadeia uma cascata na face intracelular. Esse evento é determinante na elevação na captação de glicose e direcionamento das vias metabólicas, culminado assim na formação das reservas glicogênicas. Dessa forma, foi observado que o grupo jovem controle apresentou reservas glicogênicas hepáticas similares ao descrito na literatura.

Com relação á análise da ação do picolinato de cromo nas reservas glicogênicas do grupo jovem mostrou aumento significativo tanto no fígado quanto no músculo sóleo (fibra vermelha, fibra tipo I, com maior população de receptores de insulina) indicando o sitio de ação do cromo, o qual tem sido descrito como mineral que melhora a sinalização insulínica promove elevação no número de receptores insulínicos, ativa as vias citosólicas pós-receptor insulínico elevando, com isto, a sensibilidade insulínica ${ }^{19}$.

Classicamente tem sido descrito as alterações quimio-metabólicas e funcionais que acompanham o envelhecimento ${ }^{20}$. No que se refere à funcionalidade e eficiência dos sistemas metabólicos, tem sido descrito que o músculo esquelético envelhecido apresenta modificações na sensibilidade a hormônios, redução na capacidade de metabolizar glicose, alterações nas vias pós-receptor da insulina, representadas pela redução na eficiência do triunvirato: captar glicose, metabolizar, reservar, bem como diminuir a capacidade de mobilizar suas reservas energéticas, em especial as reservas de glicogênio ${ }^{21,22}$.

Recentemente, Qiao et al. ${ }^{23}$ reiteraram que ainda não há consenso sobre o exato mecanismo ligado as ações do cromo no organismo; no entanto, alguns passos de sua sinalização já foram descritos, merecendo destacar a habilidade de modular a atividade da enzima glicogênio sintetase que regula a síntese de glicogênio, bem como o mineral interage com a enzima UCP3, estimulando a captação de glicose via translocação do GLUT 4 na célula muscular esquelética por ativação da via PI3K citosólica ${ }^{24}$. Isso sugere que o exercício físico, aliado ao uso do picolinato de cromo pode potencializar a captação de glicose via translocação do GLUT 4, fazendo com que haja um aumento da massa muscular.

\section{REFERÊNCIAS}

1. Talmadge RJ, Roy RR, Caiozzo VJ, Edgerton VR. Mechanical properties of rat soleus after long-term spinal cord transection. J Appl Physiol (1985). 2002;93(4):1487-97.

2. Reardon KA, Davis J, Kapsa RM, Choong P, Byrne E. Myostatin, insulin-like growth factor-1, and leukemia inhibitory factor mRNAs are upregulated in chronic human disuse muscle atrophy. Muscle Nerve. 2001;24(7):893-9.

3. Hilder TL, Tou JC, Grindeland RE, Wade CE, Graves LM. Phosphorylation of insulin receptor substrate-1 serine 307 correlates with JNK activity in atrophic skeletal muscle. FEBS Lett. 2003:553(1-2):63-7.

4. Tanaka $T$, Kariya $Y$, Hoshino $Y$. Histochemical study on the changes in muscle fibers in relation to the effects of aging on recovery from muscular atrophy caused by disuse in rats. J Orthop Sci. 2004;9(1):76-85

5. Davini R, Nunes CV. Alterações no sistema neuromuscular decorrentes do envelhecimento e o papel do exercício físico na manutenção da força muscular em indivíduos idosos. Rev Bras Fisioter. 2003;7(3):201-7.

6. Kauffman TL. Manual de reabilitação geriátrica. Rio de Janeiro: Guanabara Koogan; 2001.

7. Matsudo SMM. Envelhecimento e atividade física. Londrina: Midiograf; 2001

8. Deschenes MR. Effects of aging on muscle fibre type and size. Sports Med. 2004;34(12):809-24.

9. Nóbrega ACL. Freitas EV Oliveira MAB, Leitão MB, Lazzoli JK Nahas RM et al. Posicionamento Oficial da Sociedade Brasileira de Medicina do Esporte e da Sociedade Brasileira de Geriatria e Gerontologia. Atividade física e saúde do idoso. Rev Bras Med Esporte. 1999;5(6):207-11.

10. Zima T, Mestek O, Tesar V, Tesarová P, Nĕmecek K, Zák A, et al. Chromium levels in patients with internal diseases. Biochem Mol Biol Int. 1998:46(2):365-74.

11. Vincent JB. Relationship between glucose tolerance factor and low-molecular-weight chromium-binding substance. J Nutr. 1994;124(1):117-9.

12. Vincent JB. Mechanisms of chromium action: low-molecular-weight chromium-binding substance. J Am Coll Nutr. 1999;18(1):6-12.

13. Gomes MR, Rogero MM, Tirapegui J. Considerações sobre cromo, insulina e exercício físico. Rev Bras Med Esporte. 2005;11(5):262-6.

14. Wang ZQ, Zhang XH, Russell JC, Hulver M, Cefalu WT Chromium picolinate enhances skeletal muscle cellular insulin signaling in vivo in obese, insulin-resistant JCR:LA-Cp rats. J Nutr. 2006;136(2):415-20.

15. Lo S, Russell JC, Taylor AW. Determination of glycogen in small tissue samples. J Appl Physiol. 1970;28(2):234-6.

16. Rafacho A, Roma LP, Taboga SR, Boschero AC, Bosqueiro JR. Dexamethasone-induced insulin resistance is associated with increased connexin $36 \mathrm{mRNA}$ and protein expression in pancreatic rat islets. Can J Physiol Pharmacol. 2007:85(5):536-45.
Cabe salientar que, no presente estudo, foi observado um aumento nas reservas glicogênicas nos músculos envelhecidos, evento que ocorreu de forma generalizada nos diferentes tipos de músculos e com maior prevalência no músculo sóleo, indicando uma possível ação preferencial em músculos com maior porcentagem de fibras tipo I.

Frente aos dados até então demonstrados, é sugestiva a hipótese do picolinato de cromo manifestar sua ação preferencialmente sobre tecidos periféricos insulina sensíveis. Dessa forma, optou-se por avaliar a velocidade de captação de glicose por tecidos periféricos através do teste de tolerância a insulina, o qual não demonstrou que o picolinato promove diferença na velocidade de decaimento da glicemia no grupo jovem; no entanto, a mesma análise realizada no grupo envelhecido demonstrou aumento na velocidade de captação da hexose pelos tecidos periféricos, concordando com diferentes autores que verificaram elevação na sensibilidade tecidual a insulina frente à suplementação com cromo (in vivo) e na condição in vitro ${ }^{25-28}$.

Pautado nas amplas ações teciduais do cromo, há de se considerar a possibilidade de que o sinal possa ser potencializado de forma indireta, havendo uma modificação no processo secretório da insulina. Para esclarecer este fato foi realizado o teste de tolerância à glicose. Os resultados mostraram que a área sob a curva foi menor, tanto no grupo jovem quanto no grupo envelhecido, sugerindo que a ação do cromo é multifatorial, ou seja, atua enquanto secretagogo da insulina bem como potencializa a amplificação do sinal insulínico nos tecidos periféricos 29,30 .

\section{CONCLUSÃO}

O principal achado do presente estudo foi que o picolinato de cromo promoveu expressiva melhora nas reservas energéticas musculares dos animais envelhecidos, indicando que pode ser uma ferramenta farmacológica importante para a manutenção da homeostasia metabólica muscular utilizada no envelhecimento, condição onde a musculatura torna-se comprometida.

Todos os autores declararam não haver qualquer potencial conflito de interesses referente a este artigo.
17. Baynes J, Dominiczak M. Bioquímica médica. São Paulo: Manole; 2000.

18. Leto D, Saltiel AR. Regulation of glucose transport by insulin: traffic control of GLUT4. Nat Rev Mol Cell Biol. 2012;13(6):383-96.

19. Sundaram B, Singhal K, Sandhir R. Ameliorating effect of chromium administration on hepatic glucose metabolism in streptozotocin-induced experimental diabetes. Biofactors. 2012;38(1):59-68.

20. Mazo G, Lopes MA, Benedetti TB. Atividade física e o idoso: concepção gerontológica. Porto Alegre: Sulina; 2004

21. Katz MS, Lowenthal DT. Influences of age and exercise on glucose metabolism: implications for management of older diabetics. South Med J. 1994;87(5):S70-3.

22. Sumida KD, Crandall SC, Chadha PL, Qureshi T. Hepatic gluconeogenic capacity from various precursors in young versus old rats. Metabolism. 2002;51(7):876-80.

23. Qiao W, Peng Z, Wang Z, Wei J, Zhou A. Chromium improves glucose uptake and metabolism through upregulating the mRNA levels of IR, GLUT4, GS, and UCP3 in skeletal muscle cells. Biol Trace Elem Res. 2009;131(2):133-42.

24. Huppertz C, Fischer BM, Kim YB, Kotani K, Vidal-Puig A, Slieker L, et al. Uncoupling protein 3 (UCP3) stimulates glucose uptake in muscle cells through a phosphoinositide 3-kinase-dependent mechanism. J Biol Chem. 2001;276(16):12520-9.

25. Anderson RA. Chromium, glucose intolerance and diabetes. J Am Coll Nutr. 1998;17(6):548-55.

26. Yoshimoto S, Sakamoto K, Wakabayashi I, Masui H. Effect of chromium administration on glucose tolerance in stroke-prone spontaneously hypertensive rats with streptozotocin-induced diabetes. Metabolism. 1992;41(6):636-42.

27. Chen WY, Chen CJ, Liu CH, Mao FC. Chromium supplementation enhances insulin signalling in skeletal muscle of obese KK/HIJ diabetic mice. Diabetes Obes Metab. 2009;11(4):293-303.

28. Wang Y, Van Oort MM, Yao M, Van der Horst DJ, Rodenburg KW. Insulin and chromium picolinate induce translocation of CD36 to the plasma membrane through different signaling pathways in 3T3-L1 adipocytes, and with a differential functionality of the CD36. Biol Trace Elem Res. 2011;142(3):735-47.

29. Sharma S, Agrawal RP, Choudhary M, Jain S, Goyal S, Agarwal V. Beneficial effect of chromium supplementation on glucose, $\mathrm{HbA1C}$ and lipid variables in individuals with newly onset type-2 diabetes. Trace Elem Med Biol. 2011;25(3):149-53.

30. Kwon MJ, Chung HS, Yoon CS, Ko JH, Jun HJ, Kim TK, et al. The effect of chromium on rat insulinoma cells in high glucose conditions. Life Sci. 2010;87(13-14):401-4 